\title{
Exploring and Supporting Today's Collaborative Writing
}

\section{Dakuo Wang}

University of California, Irvine

Irvine, CA 92617, USA

dakuow1@uci.edu

\section{Abstract}

Collaborative writing has become increasingly common in many professions, however, when compared to the extensive research conducted 30 years ago, we know very little about it today. Now that both tools and people's skills have changed, how are people actually using these new tools in their collaborations? In my dissertation project, I investigate how people write together now, using both qualitative (i.e., interviewing participants and hand-coding co-authored documents) and quantitative (i.e., statistical modeling using Google Docs log data) research methods. I have also built

Permission to make digital or hard copies of part or all of this work for personal or classroom use is granted without fee provided that copies are not made or distributed for profit or commercial advantage and that copies bear this notice and the full citation on the first page. Copyrights for third-party components of this work must be honored. For all other for third-party components of this
uses, contact the Owner/Author.

Copyright is held by the owner/author(s).

CHI'16 Extended Abstracts, May 07-12, 2016, San Jose, CA, USA ACM 978-1-4503-4082-3/16/05.

http://dx.doi.org/10.1145/2851581.2859013 information visualization systems to complement existing research methods. By understanding what practices and tools people use in collaborative writing, I will propose design implications for system designers to improve the tools, as well as collaboration guidelines

for people to write together better.

\section{Author Keywords}

Collaborative Writing; information visualization;

computer-supported cooperative work; mixed research methods; Google Docs

\section{ACM Classification Keywords}

H.5.m. Information interfaces and presentation (e.g., HCI): Miscellaneous.

\section{Research Situation}

I am a fourth year Ph.D. candidate in the Department of Informatics at the University of California, Irvine. I passed my candidacy exam in December 2014, and I expect to finish in summer 2016. As I am part of the Hana Ohana research group, led by Gary and Judy Olson, my research focuses on $\mathrm{HCI}, \mathrm{CSCW}$ and Information Visualization. In particular, my dissertation project investigates the research topic: how people write together now. 
In my dissertation project, I have developed two information visualization systems to support our research. These systems have exhibited novel findings, and have proven useful to end-users as well. Using these systems and statistical modeling methods, I have conducted quantitative research to analyze how students collaboratively wrote 96 documents on Google Docs. In addition, I have conducted qualitative research, in which I interviewed 30 participants from various professions on how they perceived their collaborative writing practices. I am applying to the Doctoral Consortium at CHI 2016 because I believe that this consortium can provide me with a great opportunity to discuss both my dissertation work and future career plans with senior faculty and student peers.

\section{Context and Motivation}

Collaborative writing has become more common and important in many professions [7]. The goal of collaborative writing research in $\mathrm{HCI}$ is to better understand today's collaborative writing practices with the help of modernized technologies (e.g., Word and Google Docs), and to generate design implications for improving the tools that facilitate collaborative writing. Many researchers and designers had explored this topic 30 years ago $[1 ; 2 ; 6 ; 9 ; 11 ; 12]$, I believe it is critical to revisit this topic now. The two major reasons are: a) people have become more skilled with technology; and b) technologies have become more advanced. On the one hand, people's technology-readiness is much higher than before. People, even young children, are much more comfortable with computers today than they were decades ago. On the other hand, today's technologies are very different than they used to be. Many features that support collaboration (e.g., Tracking changes in Word) were not widely used then. Due to these changes, I believe people's practices in collaborative writing are significantly different from what they used to be 30 years ago. Therefore, we need to explore these practices to refresh our knowledge in order to improve the design of existing tools, and offer guidelines adapted to the new technologies and practices of collaborative writing.

\section{Related Work}

In the early 1990s, Posner and Baecker were among the first generation of researchers in Human Computer Interaction ( $\mathrm{HCI})$ who studied collaborative writing [1; $2 ; 6 ; 9 ; 11 ; 12]$. In 1992, their classic paper, "How people write together", reported the results from interviews of 10 people who had participated in 22 collaborative writing projects [11]. They developed a theoretical framework to describe various aspects of collaborative writing, which then was extended by Lowry et al. [8]. It includes: collaborative writing activities, document control modes, roles, tools, and work modes.

Very recently, a few researchers have started to revisit the collaborative writing topic. Researchers at Google conducted an internal study to investigate how its employees use Google Docs in collaborative writing [13]. They found that Google Docs is particularly usefu for new employees to become acclimated with their group. Another group of researchers studied how people edit each other's work on Google Docs and they found that co-authors are more likely to comment, rather than directly change others' words if they are not familiar with each other [3; 4]. Blau and Caspi studied how people perceive the quality of a coauthored document [5]. From an experiment with 188 
college students working in pairs, Blau and Caspi found that participants thought the others' editing lowered the documents' quality, whereas their contributions increased the documents' quality. Although each of these studies only focused on particular aspects of collaborative writing, they have presented novel findings. The fact that we are still finding new insights into collaborative writing indicates that people are writing together differently than before, and we should systematically revisit this research topic now.

\section{Problem Statement}

The main research question of this work is how people write together now. In particular, I am interested in the following questions:

- Why do people write collaboratively?

- What types of documents do people write with others versus writing alone?

- What tools and features are people using in their collaborative writing practices? And why do people prefer one tool over the others?

- What are the collaboration strategies that people use in collaborative writing, and which strategies can lead to better collaboration (i.e., collaborators can happily produce a higher quality writing in a more efficient way)?

In addition to answering these research questions, I am also interested in addressing them with new research methodologies. Previous ways of studying collaborative writing have been based on traditional qualitative and quantitative methods. But these methods may not be sufficient because in today's world, huge amount of data are generated through people writing in the cloud. Thanks to the development of Big Data techniques and the fact that today's word processors collect traces of user behavior (keystroke by keystroke), I have the opportunity to answer these questions by analyzing at scale. Therefore, I ask the additional question:

- Can I use Big Data techniques (i.e., natural language processing, text mining, and information visualization) to develop tools that can facilitate researchers of collaborative writing, and can improve co-authors' collaborating experiences as well?

\section{Research Methods}

In my dissertation work, I have conducted an in-depth interview study of collaborative writing practices of a broad user population (30 participants), including college students, faculty, industrial engineers and researchers, managers, consultants, accountants, and lawyers. In addition to the qualitative research method of relying on what people think they do, I also investigated how people actually write. I collected the data traces, which indicate both authorship and timestamps of users' writing activities, of 96 class assignment documents co-authored by 33 groups of 46 students using Google Docs. Along with my research group, I analyzed the data using hierarchical linear regression and found that certain features of collaboration (e.g., the evenness of co-authors' contributions) correlated with documents' quality [10]. In addition, by using state-of-the-art natural language processing (NLP), text mining (TM), and information visualization (InfoVis) techniques, I have developed two InfoVis systems (DocuViz [14] and AuthorViz) as a new research approach to facilitate our study.

\section{Dissertation Status}

To date, I have developed two systems, DocuViz and AuthorViz. The first system, DocuViz [14], shows a 


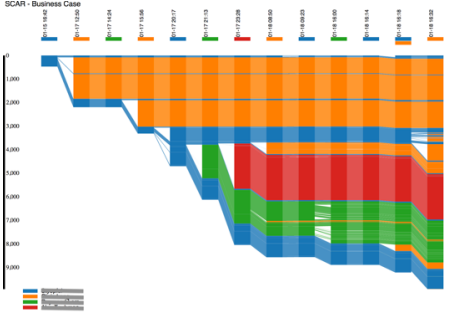

Figure 1: An example of DocuViz visualization

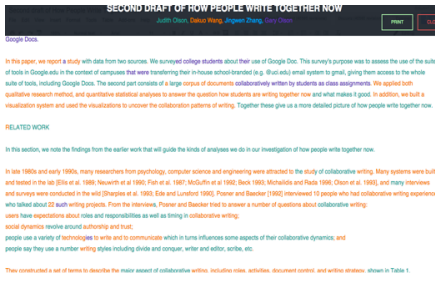

Figure 2: An example of AuthorViz visualization time-line view of how a Google Doc document evolves over time (Figure 1). The second, AuthorViz, color codes the content showing who contributed what in the final version (Figure 2).

The DocuViz and AuthorViz systems have received positive feedback from both academia and end-users. Together with my research group, I used DocuViz to analyze 96 co-authored documents collected from consenting student teams. From this analysis, we identified new patterns of writing collaborations. We have reported some findings in a peer-reviewed paper [14], and we are developing one more journal paper [10]. As for the end-users, for example, an English instructor at Hunter College, NYC, introduced DocuViz to his students of an English writing class. He found the tool to be useful in promoting students' reflective thinking, which helps them improve their writing skills. He shared with us the overall comment that "... many [students] found the maps to give them useful information about how they wrote together - they made the invisible visible ...". Our work has also attracted corporate attentions. Google invited us to give two talks to share with them what we built and found, and encouraged us to develop a more sophisticated version of the tool so that they can use it to analyze the collaborations of the writing of their internal documents.

In addition to the large-scale data-intensive research over what people actually do when they write together now, we also need in-depth qualitative work on what people think they do. Thus I have conducted an indepth interview study on a broad user population about their collaborative writing practices. I found, for example, a number of factors affect how people work and with what technology, such as occupation, scenario, personality, familiarity with the technology, and social status in the group. The study results are currently in submission at CHI 2016 as a full paper.

I am writing my dissertation to wrap up our understandings from the big data research approach, the quantitative study, and the qualitative user study. In my dissertation, I will extend the existing framework on collaborative writing, propose design implications, suggest collaboration guidelines, and embed the good practices into the tools that we developed. This will help collaborators achieve better collaborations in the sense of quality, efficiency, and the relationship between collaborators.

\section{Expected Contribution}

In my continuing analyses, I will contribute to the discussion and exploration surrounding collaborative writing and the practices that new tools enable. This research will form the core of my dissertation, and I plan to disseminate the results through papers on these topics. In addition to the scholarly products, I also plan to apply tailored research results to the design of new tools to support end-users in collaborative writing, and to contribute the tools freely to the public. On an applied level, the results and tools will help in developing new paradigms for coordinating and supporting collaborative writing practices. On a broader level, the results and tools could have implications for designing the next generation technologies related not only to collaborative writing, but also other collaborations, like software development. 


\section{Acknowledgements}

I thank all the students and interviewees who participated in our studies. I also thank all members of the Hana Ohana research group, who wrote and provided helpful comments on previous papers and this application. My work is generously supported by a Google Focused Faculty Research Award and a grant from the National Science Foundation (ACI-1322304).

\section{References}

1. Ronald M. Baecker, Nastos Dimitrios, Ilona R. Posner, and Kelly L. Mawby. 1993. The usercentered iterative design of collaborative writing software. In Proceedings of the INTERACT'93 and CHI'93, 399-405.

2. Eevi E. Beck. 1993. A survey of experiences of collaborative writing. In Computer supported collaborative writing, pp. 87-112. Springer London.

3. Jeremy Birnholtz and Steven Ibara. 2012. Tracking changes in collaborative writing: edits, visibility and group maintenance. In Proceedings of the ACM 2012 conference on Computer Supported Cooperative Work, 809-818.

4. Jeremy Birnholtz, Stephanie Steinhardt, and Antonella Pavese. 2013. Write here, write now!: an experimental study of group maintenance in collaborative writing. In Proceedings of the $\mathrm{CHI}^{\prime} 13$, 961-970.

5. Ina Blau and Avner Caspi. 2009. What type of collaboration helps? Psychological ownership, perceived learning and outcome quality of collaboration using Google Docs. In Proceedings of the Chais conference on instructional technologies research, 48-55.

6. Paul Dourish, and Victoria Bellotti. 1992.

Awareness and coordination in shared workspaces.

In Proceedings of the 1992 ACM conference on

Computer-supported cooperative work, 107-114.
7. Lisa S. Ede, and Andrea A. Lunsford. 1990. Singular texts/plural authors: Perspectives on collaborative writing. SIU Press.

8. Paul Benjamin Lowry, Aaron Curtis, and Michelle René Lowry. 2004. Building a taxonomy and nomenclature of collaborative writing to improve interdisciplinary research and practice. Journal of Business Communication 41, no. 1 (2004): 66-99.

9. Judith S. Olson, Gary M. Olson, Marianne Storrøsten, and Mark Carter. 1993. Groupwork close up: A comparison of the group design process with and without a simple group editor. ACM Transactions on Information Systems (TOIS) 11 no. 4 (1993): 321-348.

10. Judith S. Olson, Dakuo Wang, Jingwen Zhang, and Gary M. Olson. 2016. How people write together now. In submission to TOCHI.

11. Ilona R. Posner, and Ronald M. Baecker. 1992. How people write together [groupware]. In System Sciences, 1992. Proceedings of the Twenty-Fifth Hawaii International Conference on, vol. 4, 127138.

12. Mike Sharples, J. S. Goodlet, Eevi E. Beck, Charles C. Wood, S. M. Easterbrook, and Lydia Plowman. 1993. Research issues in the study of computer supported collaborative writing. In Computer supported collaborative writing, 9-28. Springer London.

13. Yunting Sun, Diane Lambert, Makoto Uchida, and Nicolas Remy. 2014. Collaboration in the cloud at Google. In Proceedings of the 2014 ACM conference on Web science, 239-240.

14. Dakuo Wang, Judith S. Olson, Jingwen Zhang, Trung Nguyen, and Gary M. Olson. 2015. DocuViz: Visualizing Collaborative Writing. In Proceedings of the 33rd Annual ACM Conference on Human Factors in Computing Systems, 1865-1874. 